\title{
UNIVALENCE CRITERIA AND QUASICONFORMAL EXTENSIONS
}

\author{
J. M. ANDERSON AND A. HINKKANEN
}

\begin{abstract}
Let $f$ be a locally univalent meromorphic function in the unit disk $\Delta$. Recently, Epstein obtained a differential geometric proof for the fact that if $f$ satisfies an inequality involving a suitable real-valued function $\sigma$, then $f$ is univalent in $\Delta$ and has a quasiconformal extension to the sphere. We give a more classical proof for this result by means of an explicit quasiconformal extension, and obtain generalizations of the result under suitable conditions even if $\sigma$ is allowed to be complex-valued and $\Delta$ is replaced by a quasidisk.
\end{abstract}

\section{INTRODUCTION AND RESULTS}

1.1. Let $f$ be a locally univalent meromorphic function in the unit disk $\Delta=$ $\{z:|z|<1\}$. There are several well-known criteria which ensure that $f$ is univalent in $\Delta$ and, possibly, has an extension to a $k$-quasiconformal mapping $\varphi$ of the extended complex plane $\overline{\mathbf{C}}$ onto itself. By this we mean that the complex dilatation $\mu(\varphi, z)=\varphi_{\bar{z}} / \varphi_{z}$ of the quasiconformal homeomorphism $\varphi$ satisfies

$$
\|\mu(\varphi, z)\|_{\infty} \leq k<1
$$

and that $\varphi(z)=f(z)$ for $z \in \Delta$. As usual, $\varphi_{z}=\frac{1}{2}(\partial \varphi / \partial x-i \partial \varphi / \partial y)$ etc., and $\|\mu\|_{\infty}=\operatorname{ess} \sup \{|\mu(\varphi, z)|: z \in \mathbf{C}\}$.

Such univalence criteria customarily involve the Schwarzian derivative of $f$ (Nehari criterion) defined by

$$
S(f, z)=\left(\frac{f^{\prime \prime}}{f^{\prime}}\right)^{\prime}(z)-\frac{1}{2}\left(\frac{f^{\prime \prime}}{f^{\prime}}(z)\right)^{2},
$$

or the logarithmic derivative $f^{\prime \prime} / f^{\prime}$ of $f^{\prime}$ (Becker criterion). The principal difficulty attached to the proof of such results is obtaining the necessary quasiconformal (from now on, we often use the abbreviation qc) extension and showing that its complex dilatation satisfies (1.1). Recently Epstein [6] has obtained a very general criterion for a locally univalent meromorphic function $f$ in $\Delta$ to have a $k$-qc extension. This result encompasses the conditions of Nehari and Becker mentioned above (see [6, p. 133]) and the methods of proof are of a differential geometric character.

Received by the editors April 4, 1989.

1980 Mathematics Subject Classification (1985 Revision). Primary 30C60.

The second author's research partially supported by the U.S. National Science Foundation. 
In this paper we provide an alternative and, we hope, more direct proof of such results by means of quasiconformal reflections. Let $D$ be a Jordan domain in $\overline{\mathbf{C}}$ with boundary $\partial D$. Following Ahlfors [1], we say that a qc reflection in $\partial D$ is a sense-reversing qc map of $\overline{\mathbf{C}}$ onto itself that takes $D$ onto $\overline{\mathbf{C}} \backslash \bar{D}$ and fixes each point on $\partial D$.

1.2. The theorem of Epstein is the following [6, Theorem 7.2, p. 126].

Theorem A. Let $f$ be meromorphic and locally univalent in $\Delta$. Suppose that there exists a real-valued function $\sigma$ in $C^{5}(\Delta)$ such that

$$
\left|\frac{\left(1-|z|^{2}\right)^{2}\left[\sigma_{z z}(z)-\sigma_{z}(z)^{2}-\frac{1}{2} S(f, z)\right]-2 \bar{z}\left(1-|z|^{2}\right) \sigma_{z}(z)}{1+\left(1-|z|^{2}\right)^{2} \sigma_{z \bar{z}}(z)}\right| \leq k,
$$

for some $k \in[0,1)$ and all $z \in \Delta$, and satisfying the following two supplementary conditions:

$$
1+\left(1-|z|^{2}\right)^{2} \sigma_{z \bar{z}}(z)>0
$$

and

$$
\left|\sigma_{z}(z)\right|\left(1-|z|^{2}\right) \leq k_{1} \max \left\{|z|,(2|z|)^{-1}\right\},
$$

for all $z \in \Delta$ and some $k_{1} \in[0,1)$. Then $f$ is univalent in $\Delta$ and has $a$ $k$-quasiconformal extension to $\overline{\mathbf{C}}$.

We remark that even though (1.2) appears to depend on $f$ only through $S(f)$, we may, by choosing $\sigma$ to depend on $f$, obtain conditions such as the Becker criterion which involve $f$ through quantities other than $S(f)$.

A special case of interest, suggested by Pommerenke, arises from taking $\sigma=\operatorname{Re} \log \varphi^{\prime}$, for some locally univalent analytic function $\varphi$. The principal condition (1.2) then becomes

$$
\left|\frac{1}{2}\left(1-|z|^{2}\right)^{2}[S(f, z)-S(\varphi, z)]+\bar{z}\left(1-|z|^{2}\right) \frac{\varphi^{\prime \prime}}{\varphi^{\prime}}(z)\right| \leq k .
$$

The Nehari criterion arises from taking $\varphi(z) \equiv z$ and the Becker criterion from taking $\varphi=f$. By the method of Löwner $k$-chains, Pommerenke [9] subsequently showed that, in this case, Theorem A remains true without the side-condition (1.4), while (1.3) is trivially satisfied since $\sigma$ is harmonic. Pommerenke made special use of the assumption that his condition involves an analytic function $\varphi$ rather than a more arbitrary function $\sigma$, and a crucial step in the proof is an application of the maximum principle. Also using the method of Löwner chains, T. Betker has recently extended Pommerenke's result to involve qc reflections other than $1 / \bar{z}$.

1.3. Let $D$ be a quasidisk in the finite complex plane $\mathbf{C}$ and let $\zeta(z)$ be a qc reflection of order 2 in $\partial D$ which is continuously differentiable in $\mathbf{C} \backslash(\partial D \cup\{\zeta(\infty)\})$. If $\infty \notin \partial D$, we assume that $1 / \zeta(z)$ is continuously differentiable at $z=\zeta(\infty)$. We write $J=J(\zeta, z)$ for the Jacobian determinant of 
$\zeta$ at the point $z$ and note that $J=\left|\zeta_{z}\right|^{2}-\left|\zeta_{\bar{z}}\right|^{2}<0$, since $\zeta$ is a reflection. (Recall that by definition, a $K$-quasidisk and a $K$-quasicircle are the images of the unit disk and the unit circle, respectively, under a qc map of $\overline{\mathbf{C}}$ onto itself whose maximal dilatation does not exceed $K$.)

Epstein proved his result first for functions $f$, meromorphic in a neighborhood of the closed unit disk $\bar{\Delta}$ and then dealt with the general case by using an approximation argument due to Ahlfors [1,2]. It is precisely this approximation method that gives rise to the supplementary conditions (1.3) and (1.4). In our result, proved by the method of qc reflections in a general quasicircle (see [1, pp. 299-300]) we also consider first the case when $f$ is sufficiently well behaved in the closure $\bar{D}$ of some quasidisk $D$.

Theorem 1. Let $D$ and $\zeta$ be as above and suppose that the function $f$ is locally univalent and meromorphic in a neighborhood of $\bar{D}$. Let $g$ be a complex-valued function in $C^{1}(D)$ such that for each $z_{0} \in \partial D$, we have

$$
\lim _{\substack{z \rightarrow z_{0} \\ z \in D}}\left\{|(z-\zeta(z)) g(z)|+|z-\zeta(z)|^{2}\left(\left|g_{z}(z)\right|+\left|g_{\bar{z}}(z)\right|\right)\right\}=0 \text {. }
$$

We further assume that each $z_{0} \in \partial D$ has a neighborhood $W$ such that $\left|\zeta_{\bar{z}}\right|$ is bounded in $W \backslash \bar{D}$. Suppose that

$$
\left|\frac{2 g \zeta_{\bar{z}}(z-\zeta)-(z-\zeta)^{2}\left\{g_{\bar{z}} \zeta_{z}+\zeta_{\bar{z}}\left[g^{2}-g_{z}+\frac{1}{2} S(f)\right]\right\}}{J-2 g \bar{\zeta}_{\bar{z}}(z-\zeta)+(z-\zeta)^{2}\left\{g_{\bar{z}} \bar{\zeta}_{z}+\bar{\zeta}_{\bar{z}}\left[g^{2}-g_{z}+\frac{1}{2} S(f)\right]\right\}}\right| \leq k,
$$

for some $k \in[0,1)$ and all $z \in D$. Then $f$ is univalent in $D$ and has $a$ $k$-quasiconformal extension $h$ to $\overline{\mathbf{C}}$ given by

$$
h(z)=f(\zeta(z))+\frac{(z-\zeta(z)) f^{\prime}(\zeta(z))}{1+(z-\zeta(z))\left\{g(\zeta(z))-\frac{1}{2} \frac{f^{\prime \prime}}{f^{\prime}}(\zeta(z))\right\}}
$$

for $z \in \overline{\mathbf{C}} \backslash \bar{D}$.

Note that $(1.8)$ is equivalent to

$$
g(z)=(z-\zeta(z))^{-1}+\frac{1}{2} \frac{f^{\prime \prime}}{f^{\prime}}(z)+f^{\prime}(z)[h(\zeta(z))-f(z)]^{-1}
$$

for $z \in D$. Hence $g\left(z_{0}\right)$ remains finite even if $f$ has a simple pole at $z=z_{0}$, at least if $h\left(\zeta\left(z_{0}\right)\right)$ is finite; and $h\left(\zeta\left(z_{0}\right)\right)$ is finite if the conclusion of Theorem 1 holds. In (1.7) the functions $\zeta, g, S(f)$ and their derivatives are, of course, evaluated at $z \in D$. We understand (1.7) to mean that the denominator does not vanish at any $z \in D$. In Theorem 1 we have assumed that $\infty \notin D$. If $\infty \in D$ then some technical problems arise that can be dealt with by using Möbius transformations. This will be done in $\S 9$.

Note that (1.6) is satisfied if the domain $D$ is bounded and if $g, g_{z}$ and $g_{\bar{z}}$ are bounded. This will be the case later on in our approximation arguments. Finally, we note that by [1, Lemma 2, p. 295] (or [7, Lemma 6.4, p. 40]), 
any quasicircle $\partial D$ admits a qc reflection $\zeta$ such that $\left|\zeta_{\bar{z}}\right|$ and hence $\left|\zeta_{z}\right|$ is bounded in $W \backslash \partial D$ for some neighborhood $W$ of $\partial D$ (or indeed in $\mathbf{C} \backslash \partial D$ if $\infty \in \partial D)$.

Remark. The condition (1.6) as well as the assumption concerning the boundedness of $\left|\zeta_{\bar{z}}\right|$, are required only to prove that the suggested qc extension $\tilde{f}$ of $f$ is indeed locally homeomorphic at each $z_{0} \in \partial D$. We mention without proof that (1.6) can sometimes be relaxed as follows. Suppose that $\zeta$ is continuously differentiable at $z_{0} \in \partial D \backslash\{\infty\}$. Then we may replace (1.6) by the assumption that

$$
\begin{gathered}
\operatorname{Re} \zeta_{z}\left(z_{0}\right)=0, \quad(\zeta-z) g \rightarrow \alpha, \\
(\zeta-z)^{2} g_{z} \rightarrow\left(1-\zeta_{z}\left(z_{0}\right)\right) \alpha, \quad(\zeta-z)^{2} g_{\bar{z}} \rightarrow-\alpha \zeta_{\bar{z}}\left(z_{0}\right)
\end{gathered}
$$

as $z \rightarrow z_{0}$ in $D$, where $\zeta, g, g_{z}$ and $g_{\bar{z}}$ are evaluated at $z$ and $\alpha=\alpha\left(z_{0}\right) \in \mathbf{C}$ with

$$
|\alpha|^{2}<1+2(\operatorname{Im} \alpha)\left(\operatorname{Im} \zeta_{z}\left(z_{0}\right)\right) .
$$

The conditions (1.10) and (1.11) guarantee that $\tilde{f}$ is locally homeomorphic at $z_{0}$. Requirements of this type are probably practical only if $\partial D$ is a very smooth curve, such as a circle or a line, so that $\zeta \in C^{1}(\mathbf{C})$. For example, in [4, p. 36], we have $D=\left\{z:\left|z-z_{1}\right|<R\right\}, \zeta(z)=z_{1}+R^{2}\left(\bar{z}-\bar{z}_{1}\right)^{-1}$, say, and we may take $g(z)=(c-1)(\zeta(z)-z)^{-1}$ for $z \in D$ where $c \in \mathbf{C}$ (cf. formula (6.8) in $\S 6$ below). Thus we have $\zeta_{z} \equiv 0$ and $\alpha=c-1$ for all $z_{0} \in \partial D$. Therefore $(1.11)$ is equivalent to $|c-1|<1$, which, as pointed out in $[4, \mathrm{p}$. 36 , is precisely the condition required there. Note that for this function $g$, the assumption (1.6) of Theorem 1 is not satisfied.

1.4. The condition (1.7) may appear awkward but, in fact, it characterizes functions $f$ having qc extensions. Note that if $f$, originally defined in $D$ and not necessarily in $\bar{D}$, has a $k_{0}$-qc extension to $\overline{\mathbf{C}}$ then $f$ also has a continuously differentiable $k$-qc extension $h$ to $\overline{\mathbf{C}}$, where $k$ may be slightly larger than $k_{0}$. Given such an $h$ we may define $g$ by (1.9). It can be verified that (1.7) is equivalent to the statement that $|\mu(h, \zeta(z))| \leq k$. This shows that, for any $f$ that admits a qc extension, there is some function $g$ in $C^{1}(D)$ such that (1.7) holds for all $z \in D$ and some $k \in[0,1)$. But note that if, for example, $f(D)$ has nonempty exterior $E$, one could start with any $k$-qc map $h$ of $\overline{\mathbf{C}} \backslash \bar{D}$ into $E$ and define $g$ by (1.9); then (1.7) holds. Thus, given $g$, we need some extra conditions such as (1.6) to guarantee that the function $h$ defined by (1.8) is actually connected to $f$. Further examples related to this will be given in $\S 7$.

If $D=\Delta$, we may take $\zeta(z)=1 / \bar{z}$. Then (1.7) simplifies to

$$
\left|\frac{\left(1-|z|^{2}\right)^{2}\left[g_{z}(z)-g(z)^{2}-\frac{1}{2} S(f, z)\right]-2 \bar{z}\left(1-|z|^{2}\right) g(z)}{1+\left(1-|z|^{2}\right)^{2} g_{\bar{z}}(z)}\right| \leq k \text {. }
$$


The function $g$ in Theorem 1 thus corresponds to $\sigma_{z}$ in Theorem A. If $D$ is the upper half plane we may take $\zeta(z)=\bar{z}$, and then (1.7) reads, with $z=x+i y$,

$$
4 y\left|\frac{-i g(z)+y\left[g_{z}(z)-g(z)^{2}-\frac{1}{2} S(f, z)\right]}{1+4 y^{2} g_{\bar{z}}(z)}\right| \leq k .
$$

Our method has not only the advantage of giving an explicit qc extension, but it shows, in addition, that in Theorem A the condition that $\sigma$ be real-valued is unnecessary and that it suffices for $\sigma$ to be in $C^{2}$ instead of $C^{5}$.

In $\S \S 3-6$ we deal with the general case for the unit disk, using approximation arguments. The case of a general quasidisk $D \subset \mathbf{C}$ is considered in $\S 8$. Unfortunately, technical difficulties constrain us to considering only quasidisks $D$ whose boundary $\partial D$ admits a qc reflection $\zeta$ that can be approximated in a certain sense sufficiently well by qc reflections $\zeta_{n}$ in $\partial D_{n}$. Here $\left\{D_{n}\right\}$ is an increasing sequence of quasidisks whose union is $D$. We do not know whether or not every quasicircle $\partial D$ admits a reflection $\zeta$ that can be so approximated.

\section{Proof of Theorem 1}

2.1. We must define a function $h$ in $D^{*}=\overline{\mathbf{C}} \backslash \bar{D}$ such that if $\tilde{f}(z)=h(z)$ for $z \in D^{*}$ and $\tilde{f}(z)=f(z)$ for $z \in \bar{D}$ then $\tilde{f}$ is a $k$-qc map of $\overline{\mathbf{C}}$ onto itself extending $f$. We set

$$
h(z)=\frac{u(\zeta(z))+(z-\zeta(z)) U(\zeta(z))}{v(\zeta(z))+(z-\zeta(z)) V(\zeta(z))}=\frac{A(z)}{B(z)},
$$

say, where $u, v, U$ and $V$ are functions, yet to be defined, in $D$. Such a definition of $h$ is analogous to a formula of Ahlfors [1, p. 299] where he made specific choices for $u, v, U$ and $V$ (see also [3]). Since $u, v, U$ and $V$ may be multiplied by the same function without changing $h$, we may, and we shall, choose $v(z) \equiv 1$. It is evident that we can write an arbitrary function $h$ in the form (2.1) as long as at least one of the functions $u, v, U$ and $V$ remains arbitrary. When $z \in \partial D$ we have $\zeta(z)=z$ and we want to have $h(z)=f(z)$. Hence we require that $u(z)=f(z)$ for $z \in \partial D$ and we make the reasonable (and permissible) choice of $u(z)=f(z)$ for all $z \in D$, leaving $U$ and $V$ still at our disposal.

A calculation now shows that

$$
\begin{aligned}
h_{z}(z) B(z)^{2}= & (U-f V)+\left(f^{\prime}-(U-f V)\right) \zeta_{z} \\
& +(z-\zeta)\left\{\zeta_{z}\left[(U-f V)_{z}+2 f^{\prime} V\right]+\bar{\zeta}_{z}(U-f V)_{\bar{z}}\right\} \\
& +(z-\zeta)^{2}\left\{\zeta_{z}\left(U_{z} V-V_{z} U\right)+\bar{\zeta}_{z}\left(U_{\bar{z}} V-V_{\bar{z}} U\right)\right\},
\end{aligned}
$$

where $\zeta=\zeta(z), \zeta_{z}$ and $\bar{\zeta}_{z}$ are evaluated at $z \in D^{*}$, and $U, V, f$ and their derivatives are evaluated at $\zeta(z)$. Similarly, we have

$$
\begin{aligned}
h_{\bar{z}}(z) B(z)^{2}= & \left\{f^{\prime}-(U-f V)\right\} \zeta_{\bar{z}} \\
& +(z-\zeta)\left\{\zeta_{\bar{z}}\left[(U-f V)_{z}+2 f^{\prime} V\right]+\bar{\zeta}_{\bar{z}}(U-f V)_{\bar{z}}\right\} \\
& +(z-\zeta)^{2}\left\{\zeta_{\bar{z}}\left(U_{z} V-V_{z} U\right)+\bar{\zeta}_{\bar{z}}\left(U_{\bar{z}} V-V_{\bar{z}} U\right)\right\} .
\end{aligned}
$$


In order to simplify (2.2) and (2.3) we set $U-f V=f^{\prime}$ and note that then $U-f V$ will be meromorphic in $D$ so that $(U-f V)_{\bar{z}} \equiv 0$. This choice of $U$-note that $V$ is still arbitrary, as we require-eliminates two types of terms in (2.2) and (2.3). The complex dilatation $\mu(h)$ of $h$ is now given by

$$
\begin{aligned}
& \mu(h, z)=\frac{h_{\bar{z}}(z)}{h_{z}(z)} \\
& \quad=\frac{(z-\zeta) \zeta_{\bar{z}}\left[f^{\prime \prime}+2 f^{\prime} V\right]+(z-\zeta)^{2}\left\{\left(f^{\prime \prime} V+f^{\prime} V^{2}-f^{\prime} V_{z}\right) \zeta_{\bar{z}}-f^{\prime} V_{\bar{z}} \bar{\zeta}_{\bar{z}}\right\}}{f^{\prime}+(z-\zeta) \zeta_{z}\left(f^{\prime \prime}+2 f^{\prime} V\right)+(z-\zeta)^{2}\left\{\left(f^{\prime \prime} V+f^{\prime} V^{2}-f^{\prime} V_{z}\right) \zeta_{z}-f^{\prime} V_{\bar{z}} \bar{\zeta}_{z}\right\}} .
\end{aligned}
$$

Given a function $g \in C^{1}(D)$ such that (1.7) holds, we set

$$
V(z)=g(z)-\frac{1}{2} \frac{f^{\prime \prime}}{f^{\prime}}(z) \text {. }
$$

Then $V \in C^{1}(D)$ so that $h$ is absolutely continuous on lines and differentiable everywhere in $D^{*}$ possibly apart from some exceptional points that will be discussed below. It is now a technical matter to conclude, using (2.4) and (2.5), that (1.7) is equivalent to the statement

$$
|\mu(h, z)| \leq k \text { when } z \neq \infty \neq f(\zeta(z)) \text { and } B(z) \neq 0 .
$$

Also $h$ is continuously differentiable at each $z \in D^{*}$, apart from the exceptions specified in (2.6), and hence, by the inverse function theorem, $h$ is locally homeomorphic at each such point.

2.2. The proof now follows a standard procedure. We show that $h$ is locally univalent at all points $z \in D^{*}$ and that $\tilde{f}$ is locally univalent at all $z \in \partial D$. By a well-known topological result, used in all known proofs of this kind of injectivity criterion (cf. [2, p. 23]), it follows that the function $\tilde{f}$ is a homeomorphism of $\overline{\mathbf{C}}$ onto itself. Now $\tilde{f}$ is ACL with $|\mu(\tilde{f}, z)| \leq k$ for a.e. $z \in D^{*}$ and $\tilde{f}$ is conformal in $D$. Moreover, the quasicircle $\partial D$ is a removable set for qc mappings that are homeomorphic in $\overline{\mathbf{C}}$. We conclude that $\tilde{f}$ is a $k$-qc mapping, and Theorem 1 will then be proved.

To show that $\tilde{f}$ is locally univalent at the exceptional points and at the boundary points $z_{0}$ of $D$, we apply the inverse function theorem to $\tilde{f}$, or to $1 / \tilde{f}$ if $\tilde{f}\left(z_{0}\right)=\infty$. If $z_{0} \in \partial D$, the assumption (1.6) implies that $\tilde{f}$ (or $1 / \tilde{f}$ ) is continuously differentiable at $z_{0}$ with $\tilde{f}_{z}\left(z_{0}\right)=f^{\prime}\left(z_{0}\right)$ and $\tilde{f}_{\bar{z}}\left(z_{0}\right)=0$. If $z_{0}=\infty \in \partial D$, we consider $\tilde{f}(1 / z)$ or $1 / \tilde{f}(1 / z)$ instead. We leave the details to the reader.

To remove the restrictions of (2.6), note first that, by assumption, we have $\infty \notin D$ so that $\zeta(z) \neq \infty$ for all $z \in D^{*}$. Suppose that $B\left(z_{0}\right)=0$ and $f\left(\zeta\left(z_{0}\right)\right) \neq \infty$, for some $z_{0} \in D^{*} \backslash\{\infty\}$. Then, for all $z$ in some neighborhood $W$ of $z_{0}$ we have

$$
A(z)=(z-\zeta(z)) f^{\prime}(\zeta(z))+B(z) f(\zeta(z)) \neq 0,
$$


since $f^{\prime}\left(\zeta\left(z_{0}\right)\right) \neq 0, \infty$. Hence $1 / h=B / A$ is well defined, finite and continuously differentiable in $W$. Since $|\mu(1 / h)|=|\mu(h)|$ we have $|\mu(1 / h)| \leq k$ in $W$. Thus $1 / h$, and hence $h$, is locally univalent in $W$. In particular $B(z)$ can vanish in $W$ only at $z=z_{0}$.

If $z_{0} \neq \infty$ but $f\left(\zeta\left(z_{0}\right)\right)=\infty$ then

$$
A_{1}(z)=\left[\zeta(z)-\zeta\left(z_{0}\right)\right] A(z) \text { and } B_{1}(z)=\left[\zeta(z)-\zeta\left(z_{0}\right)\right] B(z)
$$

have finite limits as $z \rightarrow z_{0}$ with $B_{1}\left(z_{0}\right) \neq 0$. We may then define $h\left(z_{0}\right)=$ $A_{1}\left(z_{0}\right) / B_{1}\left(z_{0}\right)$ and conclude that $h$ is locally univalent at $z_{0}$.

If $z_{0}=\infty \in D^{*}$ we proceed as above, multiplying $A$ and $B$ by $(z-\zeta(z))^{-1}$ if $f\left(\zeta\left(z_{0}\right)\right) \neq \infty$ and by $\left(\zeta(z)-\zeta\left(z_{0}\right)\right)(z-\zeta(z))^{-1}$ if $f\left(\zeta\left(z_{0}\right)\right)=\infty$, so that $h$ remains finite as $z \rightarrow z_{0}$. If it happens that $f\left(\zeta\left(z_{0}\right)\right) \neq \infty$ but $2 g\left(\zeta\left(z_{0}\right)\right)=\frac{f^{\prime \prime}}{f^{\prime}}\left(\zeta\left(z_{0}\right)\right)$ we consider $1 / h$ instead of $h$. Note that, in that case, $U\left(z_{0}\right)=f^{\prime}\left(\zeta\left(z_{0}\right)\right) \neq 0$ so that $1 / h(1 / z)$ is well defined, finite, and continuously differentiable in a neighborhood of $z=0$. In all these cases $h$ will be locally univalent at $z_{0}$ so that $h$, and hence $\tilde{f}$, has finally been shown to be locally homeomorphic everywhere. This completes the proof of Theorem 1 .

\section{APPROXIMATION METHODS}

In the standard case of the unit disk $\Delta$ the usual method of approximation is to set $f_{r}(z)=f(r z)$ for $0<r<1$ and to consider $f$ as the $\operatorname{limit}_{\lim _{n \rightarrow \infty}} f_{r_{n}}(z)$ for a suitable sequence $r_{n}$ increasing to 1 . The condition (1.7) at the point $r z$ will not, in general, imply (1.7) for the function $f_{r}$ at the point $z$, but various side-conditions ensuring this have been given by Epstein in Theorem A, following Ahlfors. We may also use these arguments [6, pp. 131-132], together with Theorem 1 to obtain Epstein's result. The point of Theorems 2 and 3 below, however, is that by using more careful approximation arguments, and applying Lemma 1 , which will be stated and proved in $\S 4$, we may further weaken the side-conditions required in addition to (1.7).

The function $f_{r}$ is meromorphic in a neighborhood of $\bar{\Delta}$ and we wish to apply Theorem 1 to conclude that $f_{r}$ is univalent in $\Delta$ and has a $k_{1}$-qc extension to $\overline{\mathbf{C}}$, for some $k_{1} \in[0,1)$. Therefore, at least for a sequence $r_{n} \rightarrow 1$ we need to find functions $g_{n}$ such that (1.7) holds for all $z \in \Delta$, with $f$ and $g$ replaced by $f_{n}$ and $g_{n}$. Here we write $f_{n}$ for $f_{r_{n}}$ and set $g_{n}(z)=r_{n} g\left(r_{n} z\right)$. However, it is not clear that (1.7) for $f$ and $g$ implies (1.7) for $f_{n}$ and $g_{n}$ and the extra conditions are imposed to guarantee this. From now on we shall use the reflection $\zeta(z)=1 / \bar{z}$ so that (1.7) becomes (1.12).

In Theorem $\mathrm{A}$ the function $\sigma_{z \bar{z}}$, corresponding to $g_{\bar{z}}$ in Theorem 1, is assumed to be real-valued. In this case we may replace (1.4) by the weaker condition

$$
\limsup _{|z| \rightarrow 1}|g(z)|\left(1-|z|^{2}\right) \leq k_{2}<1
$$


Theorem 2. Let $f$ be a locally univalent meromorphic function in $\Delta$. Suppose that (1.12) and (3.1) hold with $0 \leq k \leq k_{2}<1$ and that the complex-valued function $g \in C^{1}(\Delta)$ is such that $g_{\bar{z}}$ is real-valued. Suppose, further, that

$$
1+\left(1-|z|^{2}\right)^{2} g_{\bar{z}}(z)>0
$$

for all $z \in \Delta$. Then $f$ is univalent in $\Delta$ and has a $k$-quasiconformal extension $h$ to $\overline{\mathbf{C}}$ given by (1.8) with $\zeta(z)=1 / \bar{z}$.

We consider the approximation problem also when $g_{\bar{z}}$ is complex-valued. This case is technically complicated and it is the nature of things that we need an extra condition on $\left|g_{\bar{z}}\right|$.

Theorem 3. Let $f$ be a locally univalent meromorphic function in $\Delta$. Suppose that (1.12) holds for all $z \in \Delta$, where $g$ is a complex-valued function in $C^{1}(\Delta)$ with

$$
\limsup _{|z| \rightarrow 1}|g(z)|\left(1-|z|^{2}\right)^{2} \leq \tau .
$$

Suppose, further, that $0 \leq k+2 \tau<1$ and that

$$
\limsup _{|z| \rightarrow 1}\left|g_{\bar{z}}(z)\right|\left(1-|z|^{2}\right)^{2}<\frac{1}{2}\left(1-\frac{4 \tau^{2}\left(1+k^{2}\right)}{\left(1-k^{2}\right)^{2}}\right) .
$$

Then $f$ is univalent in $\Delta$ and has a $k$-quasiconformal extension $h$ to $\overline{\mathbf{C}}$ given by $(1.8)$ with $\zeta(z)=1 / \bar{z}$.

The condition (3.2) corresponds to (1.2) in Theorem A. By our interpretation of (1.12), the denominator is nonzero and is continuous. Thus, in Theorem 2 the left-hand side of (3.2) is always positive or always negative; for the most natural examples of $g$ it is positive. Perhaps (3.2) is redundant. We know of no examples of functions $f$ and $g$ for which the conclusion of Theorem 2 is true and (1.12) and (3.1) hold but (3.2) is violated.

In Theorem 3 note that

$$
4 \tau^{2}\left(1+k^{2}\right)<(1-k)^{2}\left(1+k^{2}\right)<(1-k)^{2}(1+k)^{2}=\left(1-k^{2}\right)^{2}
$$

so that the right-hand side of (3.4) is positive. Also note that (3.3) is a much weaker condition than (1.6) even if $\tau=0$. However, (3.4) seems unduly restrictive. If, instead of (3.3), $g$ satisfies the stronger condition

$$
\limsup _{|z| \rightarrow 1}|g(z)|\left(1-|z|^{2}\right) \leq M<\infty
$$

then (3.3) holds with $\tau=0$ and we may replace the right-hand side of (3.4) by $\frac{1}{2}$. Such a change in hypothesis is reasonable, for if Theorem 3 applies to $g$, then (3.5) holds. To see this, note that $g$ is then given by (1.9) with $\zeta(z)=1 / \bar{z}$ so that

$$
|g(z)|\left(1-|z|^{2}\right) \leq 1+\frac{1}{2}\left|\frac{f^{\prime \prime}}{f^{\prime}}(z)\right|\left(1-|z|^{2}\right)+\frac{\left|f^{\prime}(z)\right|\left(1-|z|^{2}\right)}{|h(1 / \bar{z})-f(z)|} .
$$


Now suppose that $f$ maps $\Delta$ conformally onto the simply connected domain $G$. If $\infty \notin G$ then

$$
\left|\frac{f^{\prime \prime}}{f^{\prime}}(z)\right|\left(1-|z|^{2}\right) \leq 6
$$

for all $z \in \Delta$; see [5, p. 42]. Moreover for $w \in G$ let $\lambda_{G}(w)$ denote the density of the Poincare metric in $G$ and $d(w, \partial G)$ the distance of $w$ from $\partial G$. Then by $[7$, formula $(1.4)$, p. 6$]$, we have

$$
\left|f^{\prime}(z)\right|\left(1-|z|^{2}\right)=\lambda_{G}(f(z))^{-1} \leq 4 d(f(z), \partial G)
$$

for all $z \in \Delta$. Since $h(1 / \bar{z}) \notin G$ we have $d(f(z), \partial G) \leq|h(1 / \bar{z})-f(z)|$. This proves (3.5) with $M=8$ when $\infty \notin G$. If $f$ has one pole in $\Delta$ we consider $(T \circ f)(z)$ as $|z| \rightarrow 1$ for some suitable Möbius transformation $T$ and the result follows again.

\section{A TECHNICAL LEMMA}

In the approximation arguments of $\S \S 5-8$ we need the following auxiliary result.

Lemma 1. Suppose that $0<A<C, D \geq 0,0 \leq k \leq k_{1}$, and that

$$
D+k A \leq k_{1} C \text {. }
$$

Let $B$ be a given complex number. Then

$$
D+k|A+B| \leq k_{1}|C+B|,
$$

provided that at least one of the following four conditions is satisfied:

(i) $k=k_{1}=D=0$;

(ii) $k=0<k_{1}$ and

$$
|B| \leq C-D / k_{1}
$$

(iii) $B$ is real with $-B \leq A$ and at least one of

$$
D+k A \leq k C
$$

and

$$
D+k A \leq k_{1} C+\left(k_{1}-k\right) B
$$

holds; or

(iv) $k>0,|B| \leq A$ and

$$
|B| \leq \frac{1}{2} \frac{k_{1}^{2} C^{2}-k^{2} A^{2}}{k_{1}^{2} C-k^{2} A}-\frac{D^{2}}{2} \frac{k_{1}^{2} C+k^{2} A}{\left(k_{1}^{2} C-k^{2} A\right)^{2}} .
$$

Proof. Cases (i) and (ii) are trivial and case (iii) nearly so. If (iii) holds then $A+B$ and $C+B$ are nonnegative. Then (4.2) reads $D+k(A+B) \leq k_{1}(C+B)$, which is equivalent to (4.5). Since $k \leq k_{1}$, it follows that (4.2) will also hold provided that $D+k|A+B| \leq k|C+B|$, which is equivalent to (4.4) since $-B \leq A$. 
We suppose then that (4.6) holds, with the right-hand side positive. We write $B=B_{1}+i B_{2}$ with $B_{1}$ and $B_{2}$ real. Then $|A+B|^{2}=A^{2}+|B|^{2}+2 A B_{1}$. Using a similar expression for $|C+B|^{2}$ and squaring (4.2) we obtain

$$
2 k D|A+B| \leq \alpha+2 \beta B_{1}+\gamma|B|^{2}=E_{1}(B) \text {, }
$$

say, where

$$
\alpha=k_{1}^{2} C^{2}-k^{2} A^{2}-D^{2}>0, \quad \beta=k_{1}^{2} C-k^{2} A>0, \quad \gamma=k_{1}^{2}-k^{2} \geq 0 .
$$

That $\alpha>0$ follows easily from (4.1). For (4.7) to hold we must have $E_{1}(B) \geq$ 0 . If $B=\rho e^{i \theta}$ we have

$$
E_{1}(B) \geq E_{1}(-\rho)=\alpha-2 \beta \rho+\gamma \rho^{2}=E(\rho) .
$$

Thus $E_{1}(B) \geq 0$ whenever $|B|=\rho_{0}$ if and only if $E\left(\rho_{0}\right) \geq 0$.

Squaring (4.7) we obtain

$$
\begin{aligned}
4 k^{2} D^{2}( & \left.A^{2}+\rho^{2}+2 A \rho \cos \theta\right) \\
& \leq(\alpha+\gamma \rho)^{2}+4 \beta^{2} \rho^{2} \cos ^{2} \theta+4 \beta \rho\left(\alpha+\gamma \rho^{2}\right) \cos \theta .
\end{aligned}
$$

Differentiation shows that for a fixed positive $\rho$, the function

$$
H(\theta)=\left[8 k^{2} D^{2} A \rho-4 \beta \rho\left(\alpha+\gamma \rho^{2}\right)\right] \cos \theta-4 \beta^{2} \rho^{2} \cos ^{2} \theta
$$

is maximized at $\theta=\pi$, provided that

$$
\beta\left(\alpha+\gamma \rho^{2}\right)-2 \beta^{2} \rho-2 k^{2} A D^{2} \geq 0 .
$$

Note that, if (4.10) holds, then $E(\rho) \geq 0$ and it suffices to check (4.2) when $B=-\rho$.

The right-hand side of (4.6) is equal to

$$
\left(\alpha \beta-2 k^{2} A D^{2}\right)\left(2 \beta^{2}\right)^{-1}=\rho_{1},
$$

say, and (4.10) holds if $\rho \leq \rho_{1}$. The condition (4.2) with $B=-\rho$ reads

$$
D+k(A-\rho) \leq k_{1}(C-\rho)
$$

since we are assuming, in addition, that $\rho \leq A<C$. If $k_{1}=k$ then (4.11) holds by (4.1). If $k_{1}>k$ then (4.11) holds for $\rho \leq \rho_{2}$ where

$$
\rho_{2}=\left(k_{1} C-k A-D\right)\left(k_{1}-k\right)^{-1} \text {. }
$$

Thus to show that (4.2) holds under condition (iv) it remains to show that $\rho_{1} \leq \rho_{2}$. The equation $E(\rho)=0$ has distinct positive solutions $\rho_{3}$ and $\rho_{4}$ with $\rho_{3}<\rho_{4}$ say. But by (4.1),

$$
\left(k_{1}-k\right) E\left(\rho_{2}\right)=2 D k\left\{D-k_{1} C-\left(2 k+k_{1}\right) A\right\} \leq 0 .
$$

Thus $E\left(\rho_{2}\right) \leq 0$ and so $\rho_{2} \geq \rho_{3}$. Since $E(\rho) \geq 0$ for $0 \leq \rho \leq \rho_{1}$ we have also $\rho_{1} \leq \rho_{3}$. Thus $\rho_{1} \leq \rho_{2}$, and Lemma 1 is proved. 
Remark 1 . The inequality (4.1) does not imply that $\rho_{1} \leq A$. For example, if $D=0$ and $k_{1}=k>0$ then $\rho_{1}=\frac{1}{2}(C+A)>A$. For this reason, we assume in (iv) that $\rho=|B| \leq A$. Note that if $k>0$, we always have

$$
\rho_{1} \leq \frac{1}{2}\left(k_{1}^{2} C^{2}-k^{2} A^{2}\right)\left(k_{1}^{2} C-k^{2} A\right)^{-1}<C .
$$

Remark 2. If (4.10) does not hold then $H(\theta)$ is maximal at $\theta=\theta_{1}$ where

$$
2 \beta^{2} \rho \cos \theta_{1}=2 k^{2} A D^{2}-\beta\left(\alpha+\gamma \rho^{2}\right) \text {. }
$$

Taking $\theta=\theta_{1}$ in (4.9) we obtain an inequality equivalent to

$$
\rho^{2} \beta(\beta-\gamma A) \leq \beta A(\alpha-\beta A)-(k A D)^{2},
$$

where $\beta-\gamma A=k_{1}^{2}(C-A)>0$ and

$$
\beta(\alpha-\beta A)-k^{2} A D^{2}=C\left\{\left(k_{1}^{2} C-k^{2} A\right)(C-A)-D^{2}\right\}>0 .
$$

In this case, we require that (4.12) holds and that $E(\rho) \geq 0$. Thus (4.6) might be weakened somewhat, but we do not pursue this.

\section{Proof of Theorem 2}

We consider $f_{n}(z)=f\left(r_{n} z\right)$ and $g_{n}(z)=r_{n} g\left(r_{n} z\right)$ corresponding to a sequence $\left\{r_{n}\right\}$ increasing to 1 such that $f$ has no poles on $\left\{z:|z|=r_{n}\right\}$. By Theorem 1 each $f_{n}$ will be univalent with a $k_{1}$-qc extension $\tilde{f}_{n}$ to $\overline{\mathbf{C}}$ provided that (1.12) holds with $f, g$ and $k$ replaced by $f_{n}, g_{n}$ and $k_{1}$. Note that the function $g_{n}$ certainly satisfies (1.6). The functions $\tilde{f}_{n}$ then form a normal family so that a subsequence will tend uniformly in $\overline{\mathbf{C}}$ to a limit function $F$. Clearly $F(z)=f(z)$ for $z \in \Delta$, and $F(z)=h(z)$ for $z \in \overline{\mathbf{C}} \backslash \bar{\Delta}$ where $h$ is given by (1.8). The function $F$ is, in fact, $k$-qc since $F=h$ in $\overline{\mathbf{C}} \backslash \bar{\Delta}$ and (1.12) is equivalent to $\|\mu(h)\|_{\infty} \leq k$.

For $f_{n}$ and $g_{n}$ the left-hand side of (1.12) has denominator

$$
1+\left(1-|z|^{2}\right)^{2} r_{n}^{2} g_{\bar{z}}\left(r_{n} z\right)
$$

while (3.2) applied at the point $r_{n} z$ reads

$$
1+\left(1-\left|r_{n} z\right|^{2}\right)^{2} g_{\bar{z}}\left(r_{n} z\right)>0
$$

That (5.1) is positive now follows from (5.2) since we only need to consider the case $g_{\bar{z}}\left(r_{n} z\right)<0$ and use the obvious inequality $\left(1-|z|^{2}\right)^{2} r_{n}^{2} \leq\left(1-\left|r_{n} z\right|^{2}\right)^{2}$.

We choose $\rho$ and $k_{1}$ with $0<\rho<1$ and $k \leq k_{2}<k_{1}<1$ such that

$$
2|g(z)| \leq k_{1}\left(1+\rho^{2}\right)\left(1-|z|^{2}\right)^{-1} \text { for } \rho^{2} \leq|z|<1 .
$$

This is possible by (3.1). Choose $n$ so large that $r_{n}>\rho$. Then

$$
2 r_{n}\left|z g\left(r_{n} z\right)\right| \leq k_{1}\left(1-r_{n}^{2}|z|^{4}\right)\left(1-|z|^{2}\right)^{-1}\left(1-r_{n}^{2}|z|^{2}\right)^{-1}
$$

for $|z| \geq \rho$ since for such $z$ we have

$$
1+\rho^{2} \leq 1+|z|^{2} \leq\left(1-r_{n}^{2}|z|^{4}\right)\left(1-|z|^{2}\right)^{-1} .
$$


We now set $r=r_{n}$ and define

$$
\begin{gathered}
A=r^{2}\left(1-r^{2}|z|^{2}\right)^{-2}, \quad B=r^{2} g_{\bar{z}}(r z), \quad C=\left(1-|z|^{2}\right)^{-2}, \\
D=2 r|z g(r z)|\left(1-r^{2}\right)\left(1-|z|^{2}\right)^{-1}\left(1-r^{2}|z|^{2}\right)^{-1}, \\
\alpha=r^{2}\left\{g_{z}(r z)-g(r z)^{2}-\frac{1}{2} S(f, r z)\right\}, \quad \beta=2 r^{3} \bar{z} g(r z)\left(1-r^{2}|z|^{2}\right)^{-1} .
\end{gathered}
$$

The assumption (1.12) for $f$ and $g$ at the point $r z$ states that

$$
|\alpha-\beta| \leq k|A+B| \text {. }
$$

We want to show that

$$
\left|\alpha-2 r \bar{z} g(r z)\left(1-|z|^{2}\right)^{-1}\right| \leq k_{1}|C+B|
$$

which is (1.12) for $f_{n}$ and $g_{n}$ at the point $z$, with $k$ replaced by $k_{1}$.

Following Ahlfors and Epstein we note that (5.4) implies (5.5) provided that

$$
2 r|z g(r z)|\left\{\left(1-|z|^{2}\right)^{-1}-r^{2}\left(1-r^{2}|z|^{2}\right)^{-1}\right\}+k|A+B| \leq k_{1}|C+B| .
$$

This is equivalent to (4.2), and since $B$ is real, we may use part (iii) of Lemma 1 to conclude that (5.6) holds provided that (4.5) and (4.1) are true. From (3.2) with $z$ replaced by $r z$ we obtain $B \geq-r^{2}\left(1-r^{2}|z|^{2}\right)^{-2}=-A$. Thus (4.5) and (4.1) will both hold if $D \leq k_{1}(C-A)$, which is equivalent to (5.3). Hence (1.12) is valid for $f_{n}$ and $g_{n}$ if $|z| \geq \rho$ and $r=r_{n}>\rho$. For $|z| \leq \rho$ the inequality (1.12) follows for $f_{n}$ and $g_{n}$ with a suitable $k_{1}$ by the uniform convergence of $\left\{f_{n}\right\}$. This completes the proof of Theorem 2 .

\section{Proof of Theorem 3}

6.1. The proof of Theorem 3 follows that of Theorem 2. We use the same notation but the proof is more complicated. As before, it suffices to show that (5.6), or, equivalently, (4.2) holds when $|z| \geq \rho$ and $r=r_{n}>\rho$ for a suitable $\rho \in(0,1)$. In particular, $\rho$ is chosen sufficiently close to 1 so that $(3.4)$ comes into effect and the denominator in (1.12) for $f_{n}$ and $g_{n}$ does not vanish for $|z| \geq \rho$ and $r_{n}>\rho$. We choose numbers $k_{1}, k_{2}, \lambda, \rho$ and $\nu$ such that, with the notation of Theorem 3 ,

$$
\begin{gathered}
0.4<\rho^{2}<1, \quad k+2 \tau<k_{2}<k_{1}<1, \\
0<\lambda<\nu=1-\left(k_{2}-k\right)^{2}\left(k_{1}^{2}+k^{2}\right)\left(k_{1}^{2}-k^{2}\right)^{-2},
\end{gathered}
$$

and such that, for $\rho^{2} \leq|z|<1$,

$$
\begin{gathered}
2|z g(z)| \leq\left(k_{2}-k\right)\left(1-|z|^{2}\right)^{-2}, \\
\left|g_{\bar{z}}(z)\right|\left(1-|z|^{2}\right)^{2} \leq \lambda / 2 .
\end{gathered}
$$

This is possible by (3.3) and (3.4) provided that $k_{1}$ is chosen sufficiently close to 1 and $k_{2}$ sufficiently close to $k+2 \tau$.

Since $\lambda<1$, we have by (6.3) that

$$
|B|=r^{2}\left|g_{\bar{z}}(r z)\right| \leq \lambda A / 2<A
$$


for $|z| \geq \rho$. Furthermore, an elementary calculation shows that

$$
\left(1-r^{2}\right)\left(1-r^{2}|z|^{2}\right)^{-3} \leq\left(1-|z|^{2}\right)^{-1}
$$

if $0 \leq r<1$ and $|z|^{2} \geq \rho^{2} \geq 0.4$. Hence

$$
D \leq\left(k_{2}-k\right) C
$$

and consequently $D+k A \leq D+k C \leq k_{2} C \leq k_{1} C$ so that (4.1) holds. To prove that (4.2) holds, there are two cases to consider.

Case I: $k=0$. By part (ii) of Lemma 1 we conclude that (4.2) will hold if $k_{1}|B|+D \leq k_{1} C$. But from (6.4) and (6.5) we have

$$
\left(k_{1}|B|+D\right) C^{-1} \leq k_{1} \lambda / 2+k_{2} \leq \frac{1}{2}\left(k_{1}-k_{2}^{2} k_{1}^{-1}\right)+k_{2} \leq k_{1}
$$

as required.

Case II: $k>0$. Since $|B|<A$ we deduce from part (iv) of Lemma 1 that (4.2) will be valid provided that

$$
2|B|\left(k_{1}^{2} C-k^{2} A\right)^{2}+D^{2}\left(k_{1}^{2} C+k^{2} A\right) \leq\left(k_{1}^{2} C^{2}-k^{2} A^{2}\right)\left(k_{1}^{2} C-k^{2} A\right) .
$$

By (6.4) and (6.5) this will be true if

$$
\text { (6.6) } \nu A\left(k_{1}^{2} C-k^{2} A\right)^{2}+\left(k_{2}-k\right)^{2} C^{2}\left(k_{1}^{2} C+k^{2} A\right) \leq\left(k_{1}^{2} C^{2}-k^{2} A^{2}\right)\left(k_{1}^{2} C-k^{2} A\right) \text {, }
$$

where $\nu$ is given by (6.1). This is equivalent to $f_{0}(C / A) \geq 0$ for a certain cubic polynomial $f_{0}(t)$. Using $(6.1)$ one can verify that $f_{0}(1)=0$ and $f_{0}^{\prime}(t) \geq 0$ for $t \geq 1$. Thus $f_{0}(t) \geq 0$ for all $t>1$ and in particular $f_{0}(C / A) \geq 0$. Thus (6.6) holds and Theorem 3 is proved.

6.2. Although the supplementary conditions of Theorems 2 and 3 are meaningful for all functions $g \in C^{1}(\Delta)$, they do not always give the best possible results. For example, Ahlfors [2, p. 25] obtained the criterion

$$
\left|\frac{1}{2} S(f, z)\left(1-|z|^{2}\right)^{2}-c(1-c) \bar{z}^{2}\right| \leq k|c|
$$

which guarantees that the function $f$, defined in $\Delta$, is univalent in $\Delta$ and has a $k$-qc extension to $\overline{\mathbf{C}}$. Here $c \in \mathbf{C}$ satisfies $|c-1| \leq k<1$. Now (6.7) is obtained from (1.12) by taking

$$
g(z)=(c-1) \bar{z}\left(1-|z|^{2}\right)^{-1}=(c-1)(1 / \bar{z}-z)^{-1}
$$

so that $g_{\bar{z}}(z)=(c-1)\left(1-|z|^{2}\right)^{-2}$. If $c$ is real then (3.1) and (3.2) hold if $|c-1|<1$. Hence Theorem 2 applies and (6.7) is a criterion for univalence if $|c-1|<1$ even if $|c-1|>k$. This difference arises since Ahlfors constructs $k$-qc approximating functions $f_{n}$ while we have used the fact that the $f_{n}$ may be just $k_{1}$-qc for some $k_{1}<1$ that need not be related to $k$.

If $c$ is not real we must apply Theorem 3 where (3.3) holds with $\tau=0$. Now (3.4) is valid if and only if $|c-1|<\frac{1}{2}$ so that we do not recover the result of Ahlfors in its full generality. This demonstrates the loss of generality suffered when passing to simpler but cruder conditions involving only $|g|$ and $\left|g_{\bar{z}}\right|$. 
When dealing with $g$, given by (6.8), Ahlfors in fact considers the relationship between (5.4) and (5.5) and makes use of the cancellation occurring in (1.12) between the terms $\left(1-|z|^{2}\right)^{2}\left(g_{z}(z)-g(z)^{2}\right)$ and $-2 \bar{z}\left(1-|z|^{2}\right) g(z)$. We may write (5.6) as

$$
\begin{gathered}
2 r|z g(r z)|\left(1-r^{2}\right)\left(1-|z|^{2}\right)^{-1}\left(1-r^{2}|z|^{2}\right)^{-1}+k r^{2}\left|\left(1-r^{2}|z|^{2}\right)^{-2}+g_{\bar{z}}(r z)\right| \\
\leq k_{1}\left|\left(1-|z|^{2}\right)^{-2}+r^{2} g_{\bar{z}}(r z)\right|
\end{gathered}
$$

which is Epstein's inequality (7.13) in [6, p. 132], and which indicates a more complicated connection between $g$ and $g_{\bar{z}}$. For a given function $g$ it may be best to deal with (6.9) directly to obtain a larger region of variability for the parameters involved. But our conditions (3.3) and (3.4) seem to be of the simplest available type which will make sense for all $g \in C^{1}(\Delta)$.

We remark that (6.7) guarantees that $f$ is univalent and has a $k$-qc extension to $\overline{\mathbf{C}}$ whenever $c \in \mathbf{C}$ and $|c-1|<1$. For one can verify that (6.9) holds with $g$ given by $(6.8)$ and with $k_{1}=\max \{k,|c-1|\}<1$. Thus our method yields a slight generalization of Ahlfors' result in [2]. Further, if (6.7) holds with $k=1$ and if $|c-1|<1$, then $f$ is univalent in $\Delta$ (cf. the argument in [4, p. 39]).

\section{SOME EXAMPLES}

There may be conditions of a different type from (3.1), (3.3) and (3.4) which, together with (1.12) imply that the conclusion of Theorems 2 and 3 remains valid. However some additional conditions are required, otherwise there may be no connection between the function $f$ and the function $h$ defined by (1.8). More precisely $f$ could be a totally arbitrary locally univalent meromorphic function in $\Delta$ such as $f(z)=e^{a z}$ where $a>\pi$. Even if $f$ happens to be univalent, such as $f(z)=e^{\pi z}$, it is possible that the exterior of $f(\Delta)$ has more than one component so that $f$ does not have a qc extension.

Nonetheless, if we suppose, for example, that $f(\Delta)$ has nonempty exterior, one of whose components is $G$, say, we may choose as our function $h$ an arbitrary $C^{1}$ qc mapping or even a conformal mapping of $\Delta^{*}=\overline{\mathbf{C}} \backslash \bar{\Delta}$ into $G$. Now define $g$ by (1.9). Then (1.12) will be satisfied simply because $h$ is qc, and there need be no connection whatsoever between $f$ and $h$.

The following example illustrates this in an even more striking fashion. We let $\psi_{1}(z)=\psi_{1}(x+i y)$ be the affine stretch $\psi_{1}(x+i y)=K x+i y$, for some $K>1$, and set $\psi(z)=(1+z)(1-z)^{-1}$. Define

$$
f(z)=z+z^{-1}, \quad|z| \leq 1, \quad \text { and } \quad h(z)=\chi(z)+\chi(z)^{-1}, \quad|z| \geq 1 \text {, }
$$

where $\chi=\psi^{-1} \circ \psi_{1} \circ \psi$. Then $h(z)=f(z)$ for $|z|=1$ and $h(\Delta)=f(\Delta)=$ $\overline{\mathbf{C}} \backslash[-2,2]$. Thus $f(\Delta)$ is not a Jordan domain and has no exterior so that $f$ has no qc extension. However the function $g$ of $(1.9)$ with $\zeta(z)=1 / \bar{z}$ is well defined even at the origin where $f$ has a pole. Also (1.12) holds for all $z \in \Delta$ with $k=(K-1)(K+1)^{-1}$; note that $k$ can be made arbitrarily small. 
If the right-hand side of (3.4) could be replaced by 1 (assuming, for example, in addition that (3.5) holds or that in any case (3.3) holds with $\tau=0$ ) then the resulting condition would be best possible of its kind. To see this, take $f(z)=$ $e^{\pi z}$ as above and let $h$ map $\Delta^{*}$ conformally onto a domain whose closure is a compact subset of the exterior of $f(\Delta)$. We assume that $h$ is conformal in a neighborhood of $\overline{\Delta^{*}}$. Then if $g$ is given by (1.9) with $\zeta(z)=1 / \bar{z}$, we have

$$
g_{\bar{z}}(z)\left(1-|z|^{2}\right)^{2}=-1+O(1-|z|) \quad \text { as }|z| \rightarrow 1
$$

so that $\lim \sup _{|z| \rightarrow 1}\left|g_{\bar{z}}(z)\right|\left(1-|z|^{2}\right)^{2}=1$. But $f(\Delta)$ is not even a Jordan domain, let alone a quasidisk.

One might say that the extra conditions can arise for two reasons. In Theorem 1 , we needed (1.6) to guarantee that $\tilde{f}$ is locally homeomorphic and, in particular, continuous on $\partial D$. In Theorems 2 and 3, we required (3.1)-(3.4) to ensure that approximation arguments based on the use of the triangle inequality would go through.

\section{APPROXIMABLE QUASIDISKS}

8.1. Let $D$ be a $K$-quasidisk in the finite plane $\mathbf{C}$ and let $z=\varphi(w)$ be a conformal mapping of $\Delta$ onto $D$ with $\psi=\varphi^{-1}$ as its inverse. For $z \in D$ we write $\lambda_{D}(z)=\left|\psi^{\prime}(z)\right|\left(1-|\psi(z)|^{2}\right)^{-1}$ for the density of the Poincare metric in $D$. Let $\left\{D_{n}\right\}_{n=1}^{\infty}$ be a sequence of $K_{1}$-quasidisks with $\bar{D}_{n} \subset D_{n+1} \subset D$ for all $n$, such that $D=\bigcup_{n} D_{n}$. Then, for each $r \in(0,1)$ we have $\psi\left(D_{n}\right) \supset\{w$ : $|w| \leq r\}$ for all sufficiently large $n$. We suppose that for some $K_{1}$ there are $K_{1}$-qc reflections $\zeta$ in $\partial D$ and $\zeta_{n}$ in $\partial D_{n}$ for $n \geq 1$, such that $\zeta \in C^{1}(D)$ and $\zeta_{n} \in C^{1}\left(D_{n}\right)$, and denote by $J$ and $J_{n}$ the Jacobian determinants of $\zeta$ and $\zeta_{n}$. Given $k, k_{1} \in[0,1)$ with $k \leq k_{1}$, we write

$$
E=\left|\zeta_{\bar{z}}\right|^{2}-k^{2}\left|\zeta_{z}\right|^{2}>0 \text { and } E_{n}=\left|\left(\zeta_{n}\right)_{\bar{z}}\right|^{2}-k_{1}^{2}\left|\left(\zeta_{n}\right)_{z}\right|^{2}>0 \text {. }
$$

We say that $D$ is an approximable $K$-quasidisk if the following holds:

(i) there exists $K_{1}$ and an exhaustion of $D$ by $K_{1}$-quasidisks $D_{n}$ of the type indicated above;

(ii) each $D_{n}$ has a $K_{1}$-qc reflection $\zeta_{n} \in C^{1}\left(D_{n}\right)$ of order 2 (that is, $\zeta_{n}\left(\zeta_{n}(z)\right.$ ) $=z$ ) and for each $z \in D$, we have $\zeta_{n}(z) \rightarrow \zeta(z)$ as $n \rightarrow \infty$;

(iii) for each $\delta>0$ there is $\rho \in(0,1)$ such that if $\psi\left(D_{n}\right) \supset\{w:|w| \leq \rho\}$ and $z \in D_{n}$ with $|\psi(z)|>\rho$, then, for such a point $z$,

$$
\begin{gathered}
\frac{\left|\zeta_{\bar{z}} J\right|}{E|z-\zeta(z)|^{2}} \leq(1+\delta) \frac{\left|\left(\zeta_{n}\right)_{\bar{z}} J_{n}\right|}{E_{n}\left|z-\zeta_{n}(z)\right|^{2}}, \\
\left|\frac{\zeta_{z} J k^{2}}{E(z-\zeta(z))^{2}}-\frac{\left(\zeta_{n}\right)_{z} J_{n} k_{1}^{2}}{E_{n}\left(z-\zeta_{n}(z)\right)^{2}}\right| \leq \delta \frac{\left|\left(\zeta_{n}\right)_{\bar{z}} J_{n}\right|}{E_{n}\left|z-\zeta_{n}(z)\right|^{2}}, \\
\left|\zeta_{\bar{z}}\right| \geq c>0 \text { and } c \leq\left|\left(\zeta_{n}\right)_{\bar{z}}\right| \leq c^{-1}, \\
\left|\left(\zeta_{n}\right)_{\bar{z}} J_{n} E_{n}^{-1}\left(z-\zeta_{n}(z)\right)^{-1}\right| \geq c \lambda_{D}(z),
\end{gathered}
$$

where $c$ depends on $K_{1}$ and $K$ only. 
We can always choose $\left\{\zeta_{n}\right\}$ so that (8.3) and (8.4) are satisfied (see [7, pp. 4041 , Lemmas 6.3 and 6.4]). It is not clear if such reflections $\zeta_{n}$ satisfy (8.1) and (8.2). In particular, (8.2) means that $\left|\zeta_{z}\right|$ and $\left|\left(\zeta_{n}\right)_{z}\right|$ are much smaller than $\left|\zeta_{\bar{z}}\right|$ and $\left|\left(\zeta_{n}\right)_{\bar{z}}\right|$. The conditions $(8.1)-(8.4)$ are of an arbitrary and unsatisfactory nature. They arise in applying the triangle inequality in the proof of Theorem 4 below. However it seems likely that, even if one were more careful, one would still need to know that $D$ was approximable in some sense similar to the above. A quasidisk with a sufficiently smooth boundary is approximable, but we do not know whether or not every quasidisk is.

Theorem 4. Let $D$ be an approximable $K$-quasidisk contained in $\mathbf{C}$ and suppose that $K_{1}, \zeta, \varphi, \psi$ and $\lambda_{D}$ are as defined above. Let $f$ be meromorphic and locally univalent in $D$ and suppose that $g$ is a complex-valued function in $C^{1}(D)$ satisfying (1.7) for some $k \in[0,1)$ and all $z \in D$. There is a positive number $\varepsilon$ depending only on $K$ and $K_{1}$ such that, if

$$
\limsup _{z \rightarrow \partial D}|g(z)|\left(\lambda_{D}(z)\right)^{-1}<(1-k) \varepsilon
$$

and

$$
\limsup _{z \rightarrow \partial D}\left|g_{\bar{z}}(z)\right|\left(\lambda_{D}(z)\right)^{-2}<(1-k) \varepsilon,
$$

then $f$ is univalent in $D$ and has a $k$-quasiconformal extension $h$ to $\overline{\mathbf{C}}$ given by (1.8) for $z \in \overline{\mathbf{C}} \backslash \bar{D}$.

Here $\zeta(z)$ is the qc reflection with respect to which $D$ is approximable. If $D$ is unbounded we understand $z \rightarrow \partial D$ in (8.5) and (8.6) to mean $|\psi(z)| \rightarrow 1$. We have had to replace (3.3) by the more restrictive condition (8.5), but it seems likely that $(8.5)$ could be replaced by

$$
\limsup _{z \rightarrow \partial D}|g(z)|\left(\lambda_{D}(z)\right)^{-1} \leq M<\infty .
$$

This condition would then also be necessary. For if $f$ is analytic and univalent in $D$ then a result of Osgood [8, Theorem 1, p. 450], shows that

$$
\left|\frac{f^{\prime \prime}}{f^{\prime}}(z)\right|\left(\lambda_{D}(z)\right)^{-1} \leq 8 \quad \text { for all } z \in D .
$$

Thus, if (1.9) holds, we may use the reasoning applied earlier to prove (3.5) to conclude that $(8.7)$ is valid with $M=12$, since $|z-\zeta(z)| \geq d(z, \partial D) \geq$ $\left(4 \lambda_{D}(z)\right)^{-1}$. The case when $f$ has a simple pole in $D$ is dealt with by a Möbius transformation as before.

8.2. To prove Theorem 4 we apply Theorem 1 . We show first that (1.7) holds for $\zeta=\zeta_{n}$ and $k$ replaced by $k_{1}$ for all $z \in D_{n}$. Note that since $D_{n}$ is bounded and the restrictions of $g, g_{z}$ and $g_{\bar{z}}$ to $D_{n}$ are bounded, the condition (1.6) holds. Then Theorem 1 yields, for each $n$, a $k_{1}$-qc extension $\tilde{f}_{n}$ of $f$ from 
$D_{n}$ to $\overline{\mathbf{C}}$ with $\tilde{f}_{n}(z)=h_{n}(z)$ for $z \in \overline{\mathbf{C}} \backslash \bar{D}_{n}$, where $h_{n}(z)$ is given by (1.8) with $\zeta=\zeta_{n}$. As in the proof of Theorem 2 , we see that a subsequence of $\left\{\tilde{f}_{n}\right\}$ tends to a $k_{1}$-qc mapping $F$, uniformly in $\overline{\mathbf{C}}$. Since $F(z)=f(z)$ for $z \in D$ and $F(z)=h(z)$ for $z \in \overline{\mathbf{C}} \backslash \bar{D}$, where $h$ is given by (1.8), we conclude that, in fact, $F$ is $k$-qc and Theorem 4 will be proved.

We denote the left-hand side of $(1.7)$ by $L(z)$, or by $L_{n}(z)$ if $\zeta$ is replaced by $\zeta_{n}$. For any $\rho \in(0,1)$, we have $L_{n}(z) \rightarrow L(z)$ as $n \rightarrow \infty$ uniformly for $|\psi(z)| \leq \rho$, and in particular the denominator in (1.7) is nonzero for $|\psi(z)| \leq \rho$ and sufficiently large $n$. So if $k_{1}>k$ then $\left|L_{n}(z)\right| \leq k_{1}$ for such $z$ and $n$.

With $\alpha=g(z)^{2}-g_{z}(z)+\frac{1}{2} S(f, z)$, our assumption (1.7) reads

$$
\left|\alpha+g_{\bar{z}} \frac{\zeta_{z}}{\zeta_{\bar{z}}}-\frac{2 g}{z-\zeta}\right| \leq \frac{k}{\left|\zeta_{\bar{z}}\right|}\left|\frac{J}{(z-\zeta)^{2}}-\frac{2 g \zeta_{z}}{z-\zeta}+g_{\bar{z}} \bar{\zeta}_{z}+\alpha \bar{\zeta}_{\bar{z}}\right|
$$

while we need to prove that

$$
\begin{aligned}
\mid \alpha+ & g_{\bar{z}} \frac{\left(\zeta_{n}\right)_{z}}{\left(\zeta_{n}\right)_{\bar{z}}}-\frac{2 g}{z-\zeta_{n}} \mid \\
& \leq \frac{k_{1}}{\left|\left(\zeta_{n}\right)_{\bar{z}}\right|}\left|\frac{J_{n}}{\left(z-\zeta_{n}\right)^{2}}-\frac{2 g\left(\zeta_{n}\right)_{z}}{z-\zeta_{n}}+g_{\bar{z}}\left(\bar{\zeta}_{n}\right)_{z}+\alpha\left(\bar{\zeta}_{n}\right)_{\bar{z}}\right| .
\end{aligned}
$$

Note that $\zeta_{\bar{z}} \neq 0 \neq\left(\zeta_{n}\right)_{\bar{z}}$ for $z \in D_{n}$ since the qc reflections $\zeta$ and $\zeta_{n}$ are in $C^{1}\left(D_{n}\right)$. We need to prove (8.9) only when $|\psi(z)|>\rho$ for a suitable $\rho$ and when $n$ is large enough; and if $\infty \in \overline{\mathbf{C}} \backslash \bar{D}$ we may take $\rho$ so close to 1 that $|\psi(\zeta(\infty))|<\rho$.

An elementary calculation shows that if $0<k_{0}<1$, the inequality $\left|\alpha-\alpha_{1}\right| \leq$ $k_{0}\left|\alpha-\alpha_{2}\right|$ holds if $\left|\alpha-\alpha_{3}\right| \leq R$ where $\alpha_{3}=\left(\alpha_{1}-k_{0}^{2} \alpha_{2}\right)\left(1-k_{0}^{2}\right)^{-1}$ and $R=k_{0}\left|\alpha_{1}-\alpha_{2}\right|\left(1-k_{0}^{2}\right)^{-1}$. Suppose that $\bar{\zeta}_{\bar{z}} \neq 0$ and set $k_{0}=k\left|\bar{\zeta}_{\bar{z}} / \zeta_{\bar{z}}\right|<k<1$. Then (8.8) holds if and only if $\alpha$ is contained in a closed disk $\Delta_{0}$ with centre $\beta_{0}$ and radius $R_{0}$ where

$$
\beta_{0} E=k^{2} J \zeta_{z}(z-\zeta)^{-2}-g_{\bar{z}} \zeta_{z} \bar{\zeta}_{z}\left(1-k^{2}\right)+2 g(z-\zeta)^{-1}\left(\left|\zeta_{\bar{z}}\right|^{2}-k^{2} \zeta_{z}^{2}\right)
$$

and

$$
R_{0} E=k\left|J \zeta_{\bar{z}}(z-\zeta)^{-2}-g_{\bar{z}} J-2 g(z-\zeta)^{-1} \zeta_{\bar{z}}\left(\zeta_{z}-\bar{\zeta}_{\bar{z}}\right)\right| .
$$

The same is trivially true if $\bar{\zeta}_{\bar{z}}=\overline{\left(\zeta_{z}\right)}=0$. Similarly (8.9) holds if and only if $\alpha$ lies in a closed disk $\Delta_{n}$ with centre $\beta_{n}$ and radius $R_{n}$, where $\beta_{n}$ and $R_{n}$ are defined like $\beta_{0}$ and $R_{0}$ with $\zeta, J, E$ and $k$ replaced by $\zeta_{n}, J_{n}, E_{n}$ and $k_{1}$, respectively. Thus (8.8) implies (8.9) if and only if $\Delta_{0} \subset \Delta_{n}$ for all large $n$ and this is true if and only if

$$
R_{0}+\left|\beta_{0}-\beta_{n}\right| \leq R_{n} \text {. }
$$

By (8.5) and (8.6) there is a number $\rho \in(0,1)$ such that $|g(z)|<\varepsilon_{1} \lambda_{D}(z)$ and $\left|g_{\bar{z}}(z)\right|<\varepsilon_{1}\left(\lambda_{D}(z)\right)^{2}$ for $\rho \leq|\psi(z)|<1$ where $\varepsilon_{1}=(1-k) \varepsilon$. Using these estimates we may calculate $\left|\beta_{0}-\beta_{n}\right|$ and use the triangle inequality to conclude 
that (8.10) will hold for $n$ sufficiently large and for $z \in D_{n}$ with $|\psi(z)|>\rho$, provided that

$$
\begin{aligned}
& \varepsilon_{1}\left(\lambda_{D}(z)\right)^{2}\left\{k E_{n}+k_{1} E+\left|\zeta_{z} \bar{\zeta}_{z} E_{n}\left(1-k^{2}\right)-\left(\zeta_{n}\right)_{z}\left(\bar{\zeta}_{n}\right)_{z} E\left(1-k_{1}^{2}\right)\right|\right\} \\
& +2 \varepsilon_{1} \lambda_{D}(z)\left\{2 k E_{n}\left|\zeta_{\bar{z}}(z-\zeta)^{-1} \operatorname{Im} \zeta_{z}\right|+2 k_{1} E\left|\left(\zeta_{n}\right)_{\bar{z}}\left(z-\zeta_{n}\right)^{-1} \operatorname{Im}\left(\zeta_{n}\right)_{z}\right|\right. \\
& +\mid E_{n}(z-\zeta)^{-1}\left(\left|\zeta_{\bar{z}}\right|^{2}-k^{2} \zeta_{2}^{2}\right) \\
& \left.\quad-E\left(z-\zeta_{n}\right)^{-1}\left(\left|\left(\zeta_{n}\right)_{\bar{z}}\right|^{2}-k_{1}^{2}\left(\zeta_{n}\right)_{z}^{2}\right) \mid\right\} \\
& \quad+\left|k^{2} J E_{n} \zeta_{z}(z-\zeta)^{-2}-k_{1}^{2} J_{n} E\left(\zeta_{n}\right)_{z}\left(z-\zeta_{n}\right)^{-2}\right|+k E_{n}\left|J \zeta_{\bar{z}}(z-\zeta)^{-2}\right| \\
& \leq k_{1} E\left|J_{n}\left(\zeta_{n}\right)_{\bar{z}}\left(z-\zeta_{n}\right)^{-2}\right| .
\end{aligned}
$$

It is now that the inequalities (8.1)-(8.4) in the definition of approximable quasidisks are used. There is a number $\kappa=\kappa\left(K_{1}\right) \in(0,1)$ such that $\left|\zeta_{z}\right| \leq$ $\kappa\left|\zeta_{\bar{z}}\right|$ and $\left|\left(\zeta_{n}\right)_{z}\right| \leq \kappa\left|\left(\zeta_{n}\right)_{\bar{z}}\right|$ for all $z \in D_{n}$. Dividing (8.11) by $E_{n} E$ and using (8.1)-(8.4) we see that (8.11) will hold if

$$
\begin{array}{r}
\frac{\varepsilon_{1} \lambda_{D}(z)}{1-\kappa^{2}}\left\{\left(34+\frac{2}{c^{2}}\right) \lambda_{D}(z)+\frac{8}{\left|z-\zeta_{n}(z)\right|}\right\} \\
\leq \frac{c \lambda_{D}(z)}{\left|z-\zeta_{n}(z)\right|}\left\{k_{1}-k-\delta\left(1+k+k^{2}\right)\right\}
\end{array}
$$

where $c$ is as in (8.3) and (8.4). Choosing $k_{1}=\frac{1}{2}(1+k)$ and taking $\varepsilon$ and $\delta$ small enough, with $\varepsilon$ depending on $c$ and $\kappa$ only, we can satisfy (8.12) provided that $\lambda_{D}(z) \leq c_{1}\left|z-\zeta_{n}(z)\right|^{-1}$ for some $c_{1}=c_{1}\left(K, K_{1}\right)$. This, in turn, follows from (8.3) and (8.4) since $\left|J_{n}\right| \leq E_{n}$. Theorem 4 is proved.

\section{MÖBIUS INVARIANCE PROPERTIES}

9.1. Let $f$ be meromorphic and locally univalent in a quasidisk $D$. We may write $f=f_{1} / f_{2}$ where $f_{1}$ and $f_{2}$ are linearly independent solutions of $y^{\prime \prime}+$ $\frac{1}{2} S(f) y=0$. The Ahlfors-Weill extension [3, p. 975], corresponds to the situation $g \equiv 0$ in (1.7) and is obtained by taking $u=f_{1}, v=f_{2}, U=f_{1}^{\prime}$ and $V=f_{2}^{\prime}$ in (2.1). It is an observation, due to Thurston, that for the Ahlfors-Weill extension we have, for each $z_{0} \in \overline{\mathbf{C}} \backslash \bar{D}$, that $h\left(z_{0}\right)=M\left(z_{0}\right)$. Here $h$ is given by (1.8) and $M$ is the Möbius transformation dependent on $f$ and $z_{0}$ such that

$$
M\left(\zeta_{0}\right)=f\left(\zeta_{0}\right), \quad M^{\prime}\left(\zeta_{0}\right)=f^{\prime}\left(\zeta_{0}\right)
$$

and

$$
M^{\prime \prime}\left(\zeta_{0}\right)=f^{\prime \prime}\left(\zeta_{0}\right)
$$

where $\zeta_{0}=\zeta\left(z_{0}\right) \in D$, and $\zeta$ is the qc reflection in $\partial D$. A calculation shows that the extension, given by $(1.8)$, has a similar interpretation in the general case with (9.1) unaltered but (9.2) replaced by

$$
M^{\prime \prime}\left(\zeta_{0}\right)=f^{\prime \prime}\left(\zeta_{0}\right)-2 f^{\prime}\left(\zeta_{0}\right) g\left(\zeta_{0}\right)
$$


Here $g$ is the function in $C^{1}(D)$ for which (1.7) is satisfied. This interpretation, therefore, is valid for all continuously differentiable qc extensions. We note that

$$
\frac{M^{\prime \prime}}{M^{\prime}}\left(\zeta_{0}\right)=\frac{f^{\prime \prime}}{f^{\prime}}\left(\zeta_{0}\right)-2 g\left(\zeta_{0}\right)=-2 V\left(\zeta_{0}\right)
$$

if we choose $v \equiv 1$ in (2.1). Thus $g$ and $V$ play, to some extent, the role of $f^{\prime \prime} / f^{\prime}$ in the Ahlfors-Weill extension.

9.2. Let $f, g, h$ and $D$ be as in Theorem 1, and let $M$ be a Möbius transformation. We set $F=M \circ f$ and $H=M \circ h$ so that $H$ is the natural qc extension of $F$. Since $S(F)=S(f)$, we see that $F$ satisfies (1.7) instead of $f$ with the same $g$ and $k$. A calculation shows that if we replace $f$ by $F$ on the right-hand side of (1.8) then we indeed get $H=M \circ h$ instead of $h$. Thus the formula (1.8) for the qc extension is Möbius invariant in this respect.

9.3. Let $f, g, h, D$ and $\zeta$ be as in Theorem 1 , and let $M$ be a Möbius transformation. We define $D_{1}=M^{-1}(D) \subset \overline{\mathbf{C}}$, so that $D_{1}$ is a quasidisk possibly containing infinity. Now put $\eta(w)=\left(M^{-1} \circ \zeta \circ M\right)(w)$ so that $\eta$ is a qc reflection in $\partial D_{1}, F=f \circ M$ in $D_{1}$, and

$$
G(w)=g(M(w)) M^{\prime}(w) \text { for } w \in D_{1} .
$$

A calculation shows that (1.7) is satisfied for all $w \in D_{1}$ with $f$ and $g$ replaced by $F$ and $G$. Further, then (1.8) yields the natural qc extension $H(\eta)=$ $h(M(\eta))$ for $F$, where $\eta=\eta(w) \in \overline{\mathbf{C}} \backslash \bar{D}_{1}$. This indicates another Möbius invariance property of the extension (1.8).

If $\infty \in D_{1}$, a calculation shows that $w^{2} G(w) \rightarrow \alpha, w^{3} G_{z}(w) \rightarrow-2 \alpha$ and $|w|^{4} G_{\bar{z}}(w) \rightarrow \beta$ as $w \rightarrow \infty$, for some $\alpha, \beta \in \mathbf{C}$, so that $G(w) \rightarrow 0$ rather strongly as $w \rightarrow \infty$.

We are now ready to make use of the Möbius invariance properties to extend Theorems 1 and 4 to the case when $\infty \in D$. For such a quasidisk $D$ we understand (1.7) in the extended sense that, not only does (1.7) hold for all finite $z \in D$ but that it holds also in some neighborhood of the origin with $f, g$ and $\zeta$ replaced by $f(1 / z),-z^{-2} g(1 / z)$ and $1 / \zeta(1 / z)$, respectively. This is the natural substitute for (1.7) at $z=\infty \in D$.

Theorem 5. Let the assumptions of Theorem 1 or of Theorem 4 be satisfied, except that now $\infty \in D$ and suppose that (1.7) holds in the above extended sense. Suppose, in addition, that $\zeta(1 / z)$ and $z^{-2} g(1 / z)$ are in $C^{1}(U)$ for some neighborhood $U$ of the origin and that $\zeta(1 / z)$ has nonzero Jacobian in $U$. Then $f$ is univalent in $D$ and has a $k$-quasiconformal extension $h$ to $\overline{\mathbf{C}}$ given by (1.8).

The proof of the above generalized form of Theorem 1 follows the proof of Theorem 1 given in $\S 2$. We need only show, in addition, that $\tilde{f}$ is locally 
univalent at $z_{0} \in \overline{\mathbf{C}} \backslash \bar{D}$ where $\zeta\left(z_{0}\right)=\infty$. Our assumptions imply that the extension $\tilde{f}(1 / z)$ of $f(1 / z)$ is locally univalent at the point $1 / z_{0} \in \overline{\mathbf{C}}_{D_{1}}$ where $D_{1}=M(D)$ and $M$ is the Möbius transformation $M(z)=1 / z$. This follows since $\eta\left(1 / z_{0}\right)=0 \neq \infty$ for the corresponding qc reflection $\eta=M^{-1} \circ \zeta \circ M$ in $\partial D_{1}$. Thus $\tilde{f}$ is locally homeomorphic at $z_{0}$ as required.

The proof of the extended version of Theorem 4 is similar and is omitted.

\section{REFERENCES}

1. L. V. Ahlfors, Quasiconformal reflections, Acta Math. 109 (1963), 291-301.

2. __ Sufficient conditions for quasiconformal extension, Discontinuous Groups and Riemann Surfaces (College Park, Md., 1973), Ann. of Math. Stud., no. 79, Princeton Univ. Press, Princeton, N.J., 1974, pp. 23-29.

3. L. Ahlfors and G. Weill, A uniqueness theorem for Beltrami equations, Proc. Amer. Math. Soc. 13 (1962), 975-978.

4. J. M. Anderson and A. Hinkkanen, A univalency criterion, Michigan Math. J. 32 (1985), 33-40.

5. J. Becker, Conformal mappings with quasiconformal extensions, Aspects of Contemporary Complex Analysis (D. A. Brannan and J. G. Clunie, eds.), Academic Press, London, 1980, pp. 37-77.

6. C. L. Epstein, The hyperbolic Gauss map and quasiconformal reflections, J. Reine Angew. Math. 372 (1986), 96-135.

7. O. Lehto, Univalent functions and Teichmüller spaces, Springer, Berlin, Heidelberg, and New York, 1987.

8. B. G. Osgood, Some properties of $f^{\prime \prime} / f^{\prime}$ and the Poincaré metric, Indiana Univ. Math. J. 31 (1982), 449-461.

9. Ch. Pommerenke, On the Epstein univalence criterion, Resultate Math. 10 (1986), 143-146.

Department of Mathematics, University College, Gower Street, London WC1E 6BT, UNITED KINGDOM (Current address of J. M. Anderson)

Department of Mathematics, The University of Texas at Austin, Austin, Texas 78712

Current address (A Hinkkanen): Department of Mathematics, University of Illinois, Urbana, Illinois 61801 